\title{
Waves in the cochlea and in acoustic rainbow sensors
}

\author{
Riccardo Marrocchio ${ }^{\mathrm{a}, *}$, Angelis Karlos ${ }^{\mathrm{b}}$, Stephen Elliott ${ }^{\mathrm{a}}$ \\ ${ }^{a}$ Institute of Sound and Vibration Research, University of Southampton, Highfield \\ Campus, Southampton, SO17 1BJ, UK \\ ${ }^{b}$ Department of Robotics and Mechatronics, AGH University of Science and \\ Technology, Al. A. Mickiewicza 30, Krakow, 30-059, Poland
}

\begin{abstract}
A WKB solution to the cochlear wave equation is derived, which results from the interaction between the passive dynamics of the basilar membrane and the 1D fluid coupling in the scalae, including both fluid viscosity and compressibility. The effect of various nondimensional parameters on the form of this solution is discussed. A nondimensional damping parameter and a nondimensional phase-shift parameter are shown to have the greatest influence on the response under normal conditions in the cochlea, with the fluid viscosity and compressibility only playing a minor role. It is then shown that in the case of an acoustic rainbow sensor, comprised of a discrete series of Helmholtz resonators in a duct, the governing wave equation in the continuous limit has the same form as the cochlear wave equation. The nondimensional compressibility parameter in this case is governed by the ratio of the Helmholtz resonator volume to that of the connecting duct and this parameter can be much larger than in the cochlea, and so plays a more dominant role in determining the response.
\end{abstract}

Keywords: cochlea; basilar membrane motion; fluid coupling; wave propagation; rainbow sensors

${ }^{*}$ Corresponding author

Email address: r.marrocchio@soton.ac.uk (Riccardo Marrocchio)

Abbreviations: BM, basilar membrane; WKB, Wentzel-Kramers-Brillouin; OW, oval window; RW, round window. 


\section{Introduction}

The cochlea is the part of the mammalian hearing mechanism which involves the transduction of acoustic waves to neural signals [1]. One of the main functions of the cochlea is to tonotopically separate different acoustic frequencies into different spatial positions along its length. This separation is achieved via a dispersive wave that propagates along the length of the cochlea, whose velocity drops to close to zero at different positions for different frequencies. As the wave slows down, its amplitude is inevitably increased, providing resolution of different frequency components. This cochlear wave involves the interaction of the fluid inertia in the two main fluid chambers of the cochlea, or scalae, and the dynamics of the partition that separates these two scalae, which incorporates the basilar membrane, BM, as illustrated in Fig. 1.

Although in principle it is possible for several forms of wave to propagate in the cochlea [2], the wave described above dominates the response in the passive cochlea, in which no cochlear amplifier is present [1]. The properties of propagation of the dispersive wave can be characterized by a complex wavenumber, whose real part determines the wave speed and whose imaginary part determines the attenuation of the wave.

The wave equation can be derived by combining the conservation of mass and momentum to an elemental volume of the fluid in the chambers. An approximate solution can then be obtained using the WKB method. The form of the WKB solution is derived here, and it is shown how the solution depends on a small number of nondimensional parameters, which is one of the main contributions of the paper. For the values relevant to the mammalian cochlea, the nondimensional viscosity and compressibility parameters are small and play little role in determining the form of the WKB solution, which depends much more strongly on the damping and phase-shift parameters.

The idea of rainbow trapping within the field of graded-index metamaterials was first proposed in photonics [3], and then was also introduced to disciplines of different wave types, such as plasmonics [4], seismic waves [5] and other types of elastic waves $[6,7,8,9]$. In the acoustics regime in particular, acoustic rainbow sensors $[10,11,12,13,14]$ consist of arrays of elements, whose properties vary with position, which have strong wave dispersion and can enhance and spatially separate different frequency components of an in-

cident acoustic wave. The sub-wavelength dimensions of the unit elements 
of acoustic rainbow sensors and their strongly dispersive acoustic behavior, not found in naturally occurring materials, means that they can be classified as acoustic metamaterials [15]. A common design of such sensors consists of an array of Helmholtz resonators, with spatially varying resonance frequencies, coupled via a duct. It is shown that when the size of these elements is small compared with the local wavelength, the resulting wave equation can be put in exactly the same form as that for the cochlear wave above. For a practical design of acoustic rainbow sensor, however, the nondimensional compressibility parameter in the WKB solution to the wave equation plays a much more important role than in the cochlea, and determines the width of a stop band within which the wave is evanescent, but beyond which the wave can again propagate.

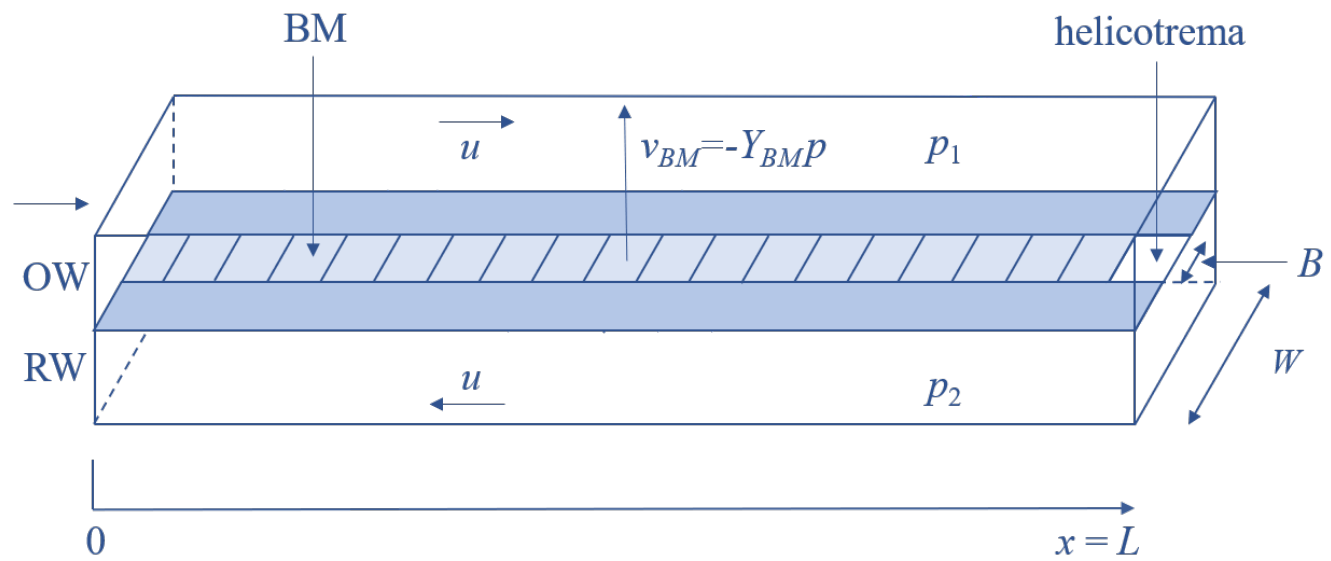

Figure 1: Schematic diagram of the box model of the uncoiled cochlea, showing the two fluid chambers, divided by the flexible basilar membrane, BM, in an otherwise rigid cochlear partition. The upper chamber is driven by the middle ear via the oval window, $\mathrm{OW}$, and the lower chamber is terminated at the basal end, $x=0$, by the flexible round window, RW, which provides pressure release. The complex pressure difference $p=p_{1}-p_{2}$ drives the locally reacting $\mathrm{BM}$ so that its velocity is $v_{B M}$, which produces equal and opposite longitudinal flow velocity $u$ in the two fluid chambers, which are connected at the apical end, $x=L$, at the helicotrema. The divisions along the BM are illustrative of the individual elements in the numerical model used to compare with the WKB solution in Section 3 . 


\section{Wavenumber in the cochlea including fluid compressibility and viscosity}

The cochlea is represented by the one-dimensional box model of Fig. 1, which is based on some simplified assumptions with respect to the real geometry. First, it is assumed that the wave in the uncoiled cochlea only propagates in one dimension, the longitudinal coordinate $x$. Secondly, it is assumed that from the point of view of the dynamics there are only two fluid chambers $[1,16,17,18,19]$. The boundaries of the cochlea are considered to be rigid and the two fluid chambers communicate with the external environment only through the membranes at the round and oval windows, at the base of the cochlea. Finally, the dynamics of the complex tissue and cell structure of the cochlear partition is simplified as a single, elastic structure, the basilar membrane, which moves in response to the difference in pressure between the two fluid chambers.

The dynamics of the system is described by a linear wave equation that is obtained using the conservation of mass and momentum applied to an elemental volume of the fluid. Assuming a time dependence of the form $\exp (\mathrm{i} \omega t)$, the continuity equation is given by:

$$
\frac{\partial u(x)}{\partial x}+\frac{\mathrm{i} \omega}{2 \rho c_{0}^{2}} p(x)=\frac{v_{B M}(x)}{h},
$$

where $u(x)$ is the longitudinal fluid velocity averaged across the cross-sectional area $(A)$ of the chamber; $p(x)$ is the pressure difference between the two scalae, averaged across the width $(W)$ of the chamber; $\rho c_{0}^{2}$ is the bulk modulus of the fluid, where $\rho$ is its density and $c_{0}$ the velocity of the sound in the fluid; $v_{B M}(x)$ is the transverse velocity of the BM, averaged across the width of the cochlea and $h$ is the effective height of the chamber, given by $h=\pi^{2} A /(8 B)$, where $B$ is the width of the basilar membrane [20]. The effective height accounts for the difference in the volumetric contribution from the assumed velocity distribution across the BM and the volume velocity associated with an entirely two-dimensional fluid model in which the whole of the cochlear partition moves with the same velocity [20]. $A, W$ and hence $h$ are assumed to be constant with $x$, giving a uniform cross section, as shown in Fig. 1.

The momentum equation is given by:

$$
\frac{\partial p(x)}{\partial x}=-2 \mathrm{i} \omega \rho u(x)-\frac{4 \nu}{h^{2}} u(x),
$$


where $\nu$ is the dynamic viscosity of the fluid. The prefactor 4 is used to be consistent with the analysis in [16], in which this term is derived by comparison with the solution of plane Poiseuille flow. Combining Eqs. (1) and (2) we obtain:

$$
\frac{\partial^{2} p(x)}{\partial x^{2}}+\frac{\omega^{2}}{c_{0}^{2}}\left(1-\frac{2 \mathrm{i} \nu}{h^{2} \omega \rho}\right) p(x)=-\frac{2 \mathrm{i} \omega \rho}{h}\left(1-\frac{2 \mathrm{i} \nu}{h^{2} \omega \rho}\right) v_{B M},
$$

which describes the relation between the velocity of the BM and the pressure distribution of the fluid. The BM is assumed to be locally reacting, so that the velocity of a point on the BM is equal to the pressure difference $p(x)$ times the opposite of the admittance $Y(x)$ of the BM, due to the sign convention:

$$
v_{B M}=-Y(x, \omega) p(x),
$$

so that Eq. (3) becomes:

$$
\frac{\partial^{2} p(x)}{\partial x^{2}}+k^{2}(x, \omega) p(x)=0,
$$

which is the wave equation of the cochlear model and $k$, the complex wavenumber, is given by:

$$
k(x, \omega)=\sqrt{\left(-\frac{2 \mathrm{i} \omega \rho}{h} Y+\frac{\omega^{2}}{c_{0}^{2}}\right)\left(1-\frac{2 \mathrm{i} \nu}{h^{2} \omega \rho}\right)} .
$$

A single-degree-of-freedom model is used for the dynamics of the passive BM, whose admittance, $Y(x)$, is given by:

$$
Y(x)=\frac{\mathrm{i} \omega}{\mathrm{i} \omega R(x)+S(x)-\omega^{2} M(x)},
$$

where $R(x), S(x)$ and $M(x)$ are, respectively, the resistance, stiffness and mass per unit area of the BM. The term $M(x)$ includes a contribution from the entrained mass of the fluid [19], and is assumed to be constant $\left(m_{0}\right)$. The longitudinal variation of the remaining variables is given by:

$$
\begin{aligned}
& R(x)=\frac{m_{0} w_{n}(x)}{Q}, \\
& S(x)=\omega_{n}^{2}(x) m_{0},
\end{aligned}
$$


where $Q$ is the quality factor of the BM local resonance, also assumed to be independent of $x$, and $\omega_{n}(x)$ is its natural angular frequency at position $x$, and this is assumed to vary exponentially along the cochlea [1], so that:

$$
\omega_{n}(x)=\omega_{b} \exp (-x / l)
$$

where $\omega_{b}$ is the natural angular frequency at the base of the cochlea and $l$ is a characteristic length. The expression of the wavenumber in Eq. (6) then becomes:

$$
k=\sqrt{\left(\frac{2 \rho \omega^{2}}{h m_{0}} \frac{1}{\left(\omega_{n}^{2}-\omega^{2}+\frac{\mathrm{i} \omega \omega_{n}}{Q}\right)}+\frac{\omega^{2}}{c_{0}^{2}}\right)\left(1-\frac{2 \mathrm{i} \nu}{h^{2} \omega \rho}\right)} .
$$

By moving the factor of $\left(1-\frac{2 \mathrm{i} \nu}{h^{2} \omega \rho}\right)$ outside the square root and simplifying the remaining term, the wavenumber can be expressed in the more insightful form:

$$
k=k_{f c}\left[\frac{G^{2} \omega_{n}^{2}+\mathrm{i} \frac{\omega \omega_{n}}{Q}-\omega^{2}}{\omega_{n}^{2}+\mathrm{i} \frac{\omega \omega_{n}}{Q}-\omega^{2}}\right]^{1 / 2},
$$

where $k_{f c}$ is the complex wavenumber in the fluid, which takes into account the effect of viscosity, and is given by:

$$
k_{f c}=\frac{\omega}{c_{0}} \sqrt{1-2 \mathrm{i} V^{2}}
$$

and $G$ is the bandgap ratio given by:

$$
G=\sqrt{1+\frac{16 N^{2}}{C_{n}^{2}}}
$$

which is the ratio of the limiting frequencies of the corresponding stop band, as shown in Fig. 2. In Eqs. (12) and (13) we have introduced the following nondimensional variables:

$$
\begin{aligned}
& C=\frac{\omega l}{c_{0}}, \\
& V=\frac{\delta}{h},
\end{aligned}
$$




$$
\begin{aligned}
& N=\frac{l}{4 h \sqrt{\mu}}, \\
& \mu=\frac{m_{0}}{2 \rho h},
\end{aligned}
$$

where $C$ is the nondimensional compressibility, equal to $2 \pi$ times the characteristic length $l$ divided by the acoustic wavelength; since $C$ depends on the excitation frequency the parameter $C_{n}$, which is $\omega_{n} l / c_{0}$, is also used and is called nondimensional compressibility at resonance; $V$ is the nondimensional viscosity which is the ratio of the viscous boundary layer thickness, $\delta$, given by $\sqrt{\nu / \omega \rho}$, to the effective height of the fluid chambers, $h ; N$ is a constant introduced in [17], called the phase-shift parameter and $\mu$ is the ratio of the mass per unit area of the BM and of the fluid in the chambers. The geometrical and physical values assumed for the cochlear model are listed in Table 1, and the corresponding nominal values of the nondimensional parameters $\left(Q_{0}\right.$, $N_{0}, C_{0}$ and $V_{0}$ ), for an excitation frequency of $1 \mathrm{kHz}$, are listed in Table 2.

Table 1: Assumed geometrical and physical values of the one-dimensional cochlear box model [20].

\begin{tabular}{lcr}
\hline Parameter & Symbol & Value \\
\hline Length of the cochlea & $L$ & $3.5 \times 10^{-2} \mathrm{~m}$ \\
Characteristic length & $l$ & $7.0 \times 10^{-3} \mathrm{~m}$ \\
Base natural frequency & $\omega_{b} / 2 \pi$ & $2.0 \times 10^{4} \mathrm{~Hz}$ \\
Effective height of a fluid chamber & $h$ & $4.1 \times 10^{-3} \mathrm{~m}$ \\
Density of the fluid & $\rho$ & $1 \times 10^{3} \mathrm{~kg} / \mathrm{m}^{3}$ \\
Mass per unit area of the BM & $m_{0}$ & $3 \times 10^{-1} \mathrm{~kg} / \mathrm{m}^{2}$ \\
Coefficient of viscosity & $\nu$ & $8.9 \times 10^{-6} \mathrm{~Pa} \mathrm{~s}$ \\
Speed of sound & $c_{0}$ & $1.5 \times 10^{3} \mathrm{~m} / \mathrm{s}$ \\
\hline
\end{tabular}

The real and imaginary parts of the wavenumber are plotted in Fig. 2a, as a function of normalized excitation frequency, for different values of the nondimensional compressibility parameter at resonance, $C_{n}$. For a nominal value of $C_{n}$, equal to $C_{0}$, a pass band is seen up to the natural frequency, for which $\omega / \omega_{n}=1$, where the real part of the wavenumber is rising and the imaginary part is for the most part very small. The rising value of the real part of the wavenumber, with an increasing slope, also means that the 
Table 2: Nominal values of nondimensional parameters in the cochlear model corresponding to an excitation frequency of $1 \mathrm{kHz}$.

\begin{tabular}{lcr}
\hline Parameter & Symbol & Value \\
\hline Quality factor & $Q_{0}$ & 5 \\
Nondimensional viscosity & $V_{0}$ & $2.9 \times 10^{-3}$ \\
Nondimensional compressibility & $C_{0}$ & $2.1 \times 10^{-2}$ \\
Phase-shift parameter & $N_{0}$ & 2.23 \\
\hline
\end{tabular}

phase and group velocities, given by $c_{p h}=\omega / k$ and $c_{g r}=\frac{\partial \omega}{\partial k}$, decrease along this band, leading to the wave being slowed so that its amplitude is concentrated near the natural frequency. There is little loss well below the natural frequency since the imaginary part of the wavenumber is small over most of this pass band. The imaginary part of the wavenumber starts to increase in magnitude at a frequency slightly before the peak of the real part. This then leads to a stop band, where the imaginary part of the wavenumber remains high in absolute terms and the wave is greatly attenuated. Only for values of $C_{n}$ much larger than the nominal one, is the value of $G \omega_{n}$, defined in Eq. (11), within the range of audible frequencies. In this case, as can be seen in Fig. 2a, beyond the stop band, a second pass band occurs, in which the wave again begins to propagate. The very high value of $C_{n}=304 C_{0}$ is chosen to compare with the rainbow sensor example below.

In Fig. 2b the real and imaginary parts of the wavenumber are plotted for different values of the nondimensional viscosity parameter $V$. Only for values of $V$ that are very much larger than the nominal value do the real and imaginary part of the wavenumber change significantly. In particular, the imaginary part increases and is nonzero even at the lowest frequencies, indicating a greater attenuation as $V$ is increased.

In Fig. 2c the real and imaginary parts of the wavenumber are plotted for different values of the quality factor $Q$. The main effect is near the peaks of the real and imaginary parts, around the natural frequency. In particular, the peaks become sharper and shift slightly to higher frequencies for higher values of $Q$. The peak of the real part of the wavenumber occurs slightly before $\omega / \omega_{n}=1$, whereas the imaginary part of $k$ becomes very large in magnitude right after $\omega / \omega_{n}=1$. The increase of $\Re\{k\}$ just before $\omega / \omega_{n}=1$ corresponds to a decrease of the phase velocity $c_{p h}$, whereas the increase slope of $\Re\{k\}$ corresponds to a decrease of the group velocity $c_{g r}$. Beyond 
$\omega / \omega_{n}=1$, the higher value of $\Im\{k\}$ corresponds to a more abrupt dissipation of the response.

In Fig. 2d the real and imaginary parts of the wavenumber are plotted for different values of the nondimensional phase-shift parameter $N$. As this parameter increases, the magnitude of the real part increases, and thus the phase speed at a given frequency decreases, up to $\omega / \omega_{n}=1$. The magnitude of the imaginary part and thus the attenuation of the wave, also increases beyond $\omega / \omega_{n}=1$.
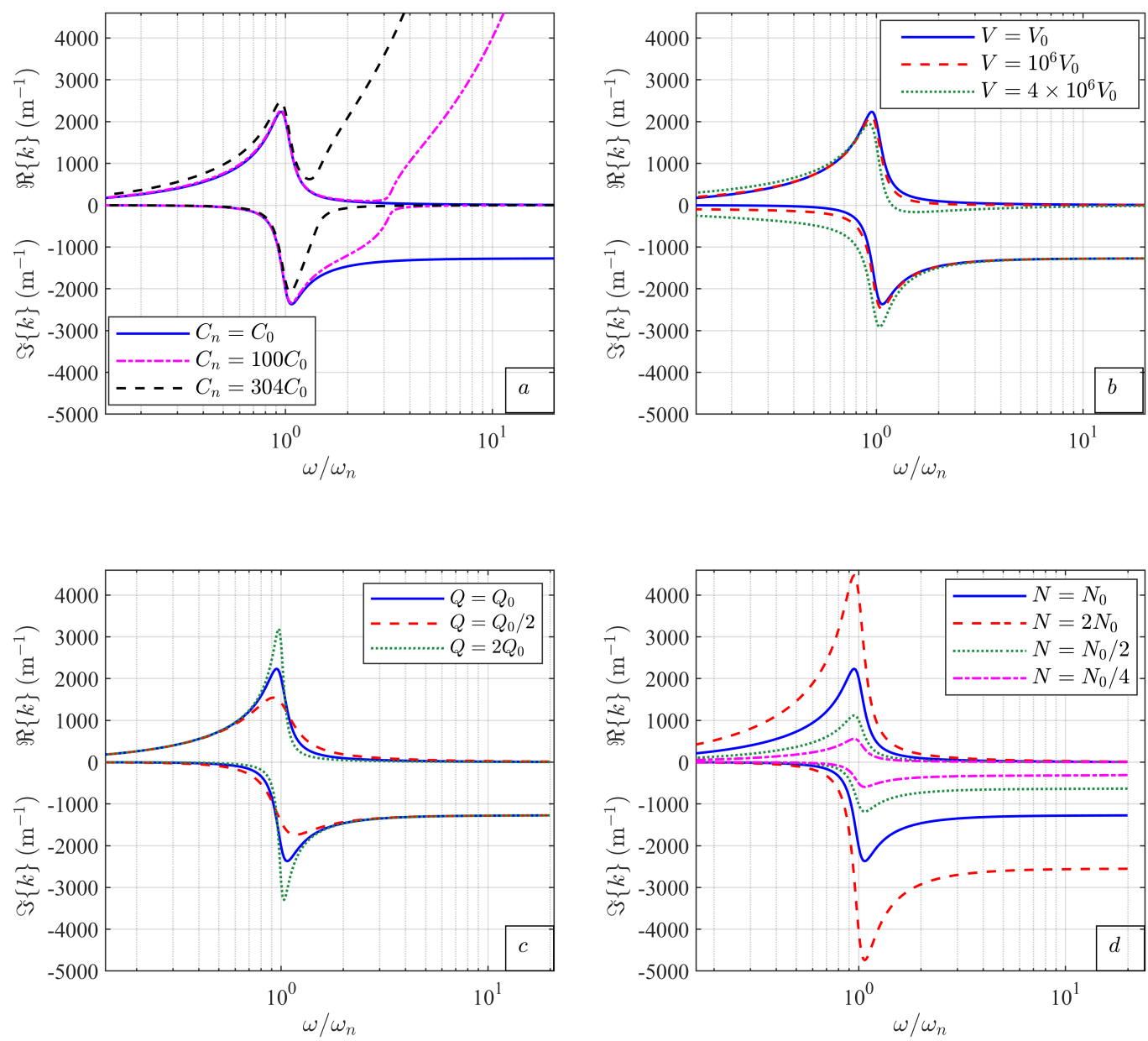

Figure 2: Variation of the amplitude (top) and of the phase (bottom) of the wavenumber as a function of normalized frequency, for (a) different values of the nondimensional compressibility parameter at resonance $C_{n}$, (b) normalized viscosity $V$, (c) quality factor $Q$ and (d) normalized phase-shift parameter $N$. 


\section{Analytic WKB solutions including compressibility and viscosity}

We now use the WKB method to derive an approximation to the exact solution of the wave equation [Eq. (5)] in terms of the nondimensional variables defined in Eqs. (14). The WKB method has been previously used to obtain closed-form solutions for 1D [17], 2D [18] and 3D [21, 22] cochlear models. These solutions have been obtained in the case of an incompressible and inviscid fluid, by assuming light damping in the BM. In [16] a semi-analytic solution is derived for the 1D case, including fluid compressibility and viscosity and with no approximation to the BM's damping. In this paper, we derive a WKB solution in terms of a number of nondimensional parameters and then discuss their contribution to the amplitude and phase response of the BM.

The ideal condition for validity of the WKB approximation is that [23, Ch.1.4]:

$$
\frac{\left|k^{\prime}(x)\right|}{\left|k^{2}(x)\right|} \ll 1 .
$$

where $k^{\prime}(x)$ is the derivative of $k$ with respect to $x$. The left hand side of this condition is plotted as a function of normalized frequency in Fig. 3 for the nominal parameters listed in Table 1, which shows that the condition is not always satisfied, particularly at low frequencies. Figure 4 shows a comparison of the WKB solution in this case with a numerical solution to a model with 512 elements, which uses finite difference approximations for the spatial derivative in Eq. (3) [20]. The approximate WKB solution differs from this numerical solution to the governing equations only for very low frequencies, as previously noted in [19]. Differences have also been noted for frequencies just above the natural frequency, where the WKB response drops off more quickly than the exact solution [24, 22, 18]. Although an assumption made in the WKB method is that only a single wave is propagating, the effects just above the natural frequency may be due to low amplitudes of an additional evanescent fluid wave $[2,25]$. Apart from these small differences, the WKB solution and the finite difference solutions are very similar around the characteristic frequency, which is the main region of interest in the study. The form of this response, with an increasing magnitude and falling phase up to the characteristic frequency, after which there is a rapid drop in amplitude with little additional phase shift, is consistent with that measured in the 
mammalian cochlea using laser methods (for example in [26]) and more recent optical coherence tomography techniques, as in [27].

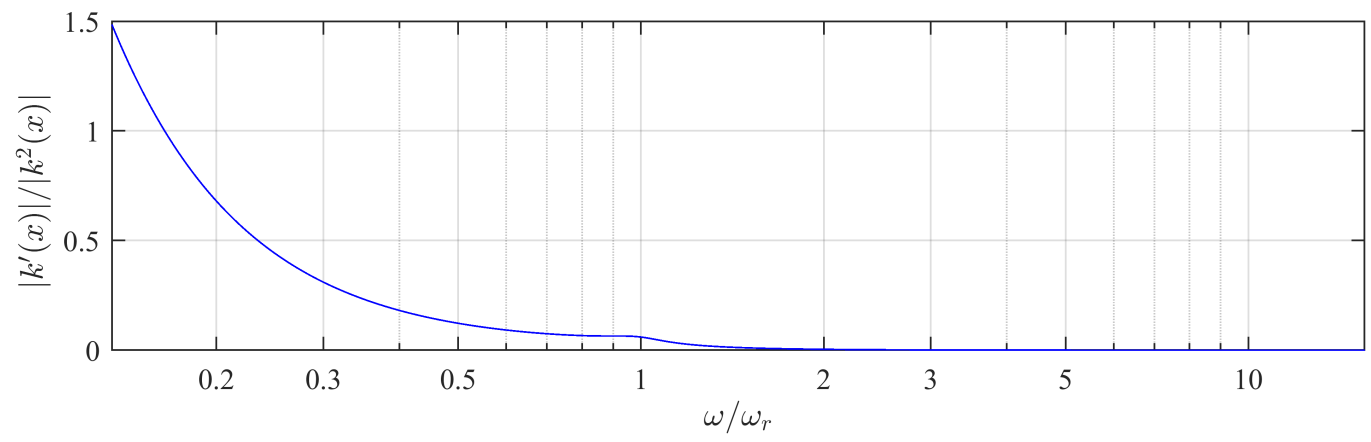

Figure 3: WKB condition as a function of normalized frequency for the nominal parameters listed in Table 1.
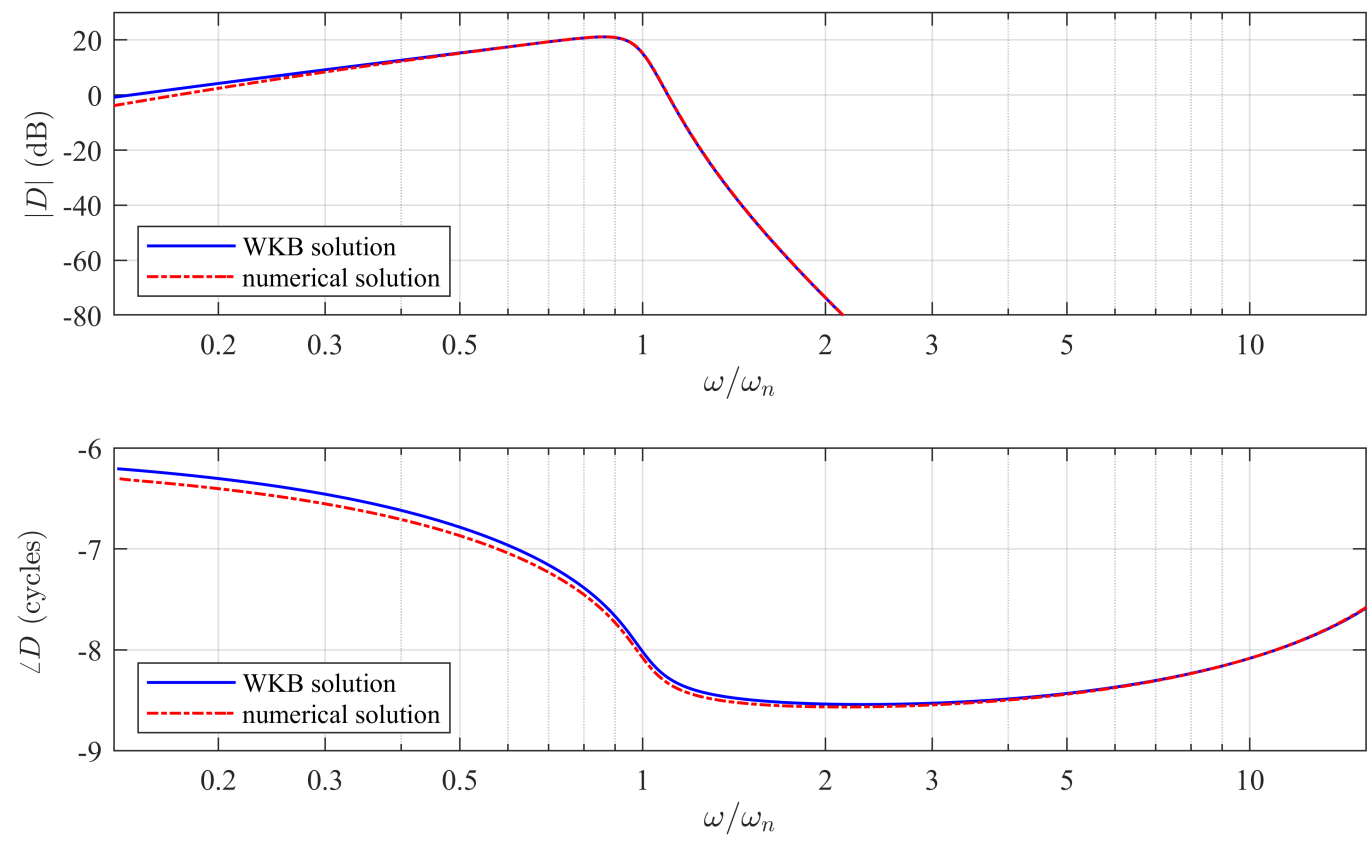

Figure 4: Comparison between the amplitude (top) and the phase (bottom) of the normalized BM displacement, obtained using the WKB method (solid line) and the elemental model (dashed line) in [20]. 
The general WKB solution to Eq. (5), with the boundary condition $p(x=$ $L)=0$, is given by:

$p(x)=\frac{p(0) \sqrt{k(0)}}{\sqrt{k(x)}} \frac{\exp \left(-\mathrm{i} \int_{0}^{x} k\left(x^{\prime}\right) \mathrm{d} x^{\prime}\right)+\exp \left(-2 \mathrm{i} \int_{0}^{l} k\left(x^{\prime}\right) \mathrm{d} x^{\prime}+\mathrm{i} \int_{0}^{x} k\left(x^{\prime}\right) \mathrm{d} x^{\prime}\right)}{1-\exp \left(-2 \mathrm{i} \int_{0}^{l} k\left(x^{\prime}\right) \mathrm{d} x^{\prime}\right)}$.

The first term in the numerator represents a forward-traveling wave, which propagates from base to apex, while the second term describes a wave that originates by reflection of the first wave at the helicotrema $(x=L)$ and then propagates towards the base. This last term is significant only for frequencies so low that the pressure $p(x)$ at the apex retains an appreciable value. It has been shown, by numerical integration of the WKB solution [16], that this reflected wave is much smaller than the forward-traveling wave and that it is important only in a region very near the apex. The WKB approximation in Eq. (15) also breaks down close to the characteristic frequency when the damping in the BM is very small, so that the peak in the coupled response is very large. It might be expected that the rapid change in the BM admittance in this case would generate a reflected wave, but it has been shown that this does not occur [28]. In practice, however, the mechanical parameters of the BM are not as smoothly varying as is represented in Eq. (8), and this roughness can generate low amplitude reflected waves, which can give rise to otoacoustic emissions [29], although these are not considered here.

We can thus simplify the solution in Eq. (16) as:

$$
p(x)=\frac{p(0) \sqrt{k(0)}}{\sqrt{k(x)}} \exp (\phi)
$$

where

$$
\phi=-\mathrm{i} \int_{0}^{x} k\left(x^{\prime}\right) \mathrm{d} x^{\prime} .
$$

The integral above can be solved as outlined in the Appendix. A simplified expression can then be obtained by making the approximation $\omega \ll \omega_{b}$, since we are interested in the form of the solution for excitation frequencies that are much lower that the natural frequency at the base. In this case, $\phi$ can 
be written as:

$$
\begin{aligned}
& \phi=\mathrm{i} C \sqrt{1-2 \mathrm{i} V}\left[\frac{1}{Q \sqrt{-1+\frac{16 N^{2}}{C^{2}}}} \mathrm{~F}\left(-\arcsin \left(\mathrm{i} / Q+\omega_{n} / \omega\right), \frac{1}{\sqrt{1-\frac{16 N^{2}}{C^{2}}}}\right)\right. \\
& +\frac{4 \omega^{2}}{Q} \sqrt{-1+\frac{16 N^{2}}{C^{2}}} \Pi\left(-Q^{2} ;-\arcsin \left(\mathrm{i} / Q+\omega_{n} / \omega\right), \frac{1}{\sqrt{1-\frac{16 N^{2}}{C^{2}}}}\right) \\
& +\operatorname{arctanh}\left(\frac{\sqrt{\left(\mathrm{i} / Q+\omega_{n} / \omega\right)^{2}-1}}{\sqrt{16 N^{2} / C^{2}-1+\left(\mathrm{i} / Q+\omega_{n} / \omega\right)^{2}}}\right) \\
& \left.+\sqrt{-1+\frac{16 N^{2}}{C^{2}}} \arctan \left(\frac{\sqrt{16 N^{2} / C^{2}-1} \sqrt{\left(\mathrm{i} / Q+\omega_{n} / \omega\right)^{2}-1}}{\sqrt{16 N^{2} / C^{2}-1+\left(\mathrm{i} / Q+\omega_{n} / \omega\right)^{2}}}\right)\right],
\end{aligned}
$$

where $\mathrm{F}$ and $\Pi$ are, respectively, an elliptic integral of the first and third kind. It is interesting to note that although the wavenumber in Eq. (11) depends on $C_{n}=\omega_{n} l / c_{0}$, via the term $G$ in Eq. (13), the phase term $\phi$ only depends on the nondimensional compressibility term $C=\omega l / c_{0}$ defined in Eq. (14a) where $\omega$ is the excitation frequency, since the term $\omega_{n}$ cancels out in the integration in Eq. (A.3).

From the pressure distribution [Eq. (17)] we can derive the velocity of a point of the BM using Eq. (4) and, from this, the corresponding displacement $\Delta$ by dividing by $\mathrm{i} \omega$, obtaining:

$$
\Delta(x, \omega)=-\frac{\sqrt[4]{1+\frac{16 N^{2}}{C^{2}\left(\frac{\omega_{b}^{2}}{\omega^{2}}+\frac{1 \omega_{b}}{Q_{0} \omega}-1\right)}}}{m_{0} \omega^{2}\left(\frac{\mathrm{i} \omega_{n}}{Q_{0} \omega}+\frac{\omega_{n}^{2}}{\omega^{2}}-1\right) \sqrt[4]{1+\frac{16 N^{2}}{C^{2}\left(\frac{i \omega_{n}}{Q_{0} \omega}+\frac{\omega_{n}^{2}}{\omega^{2}}-1\right)}}} p(0) \exp (\phi) .
$$

It is customary to normalize the displacement of the BM with respect to the displacement of the stapes, $\Delta_{s}$, which can be derived from the fluid volume velocity $u$ at $x=0$, given by Eq. (2), as:

$$
\Delta_{s}=-\frac{\left.u\right|_{x=0}}{\mathrm{i} \omega}=-\frac{\sqrt{1+\frac{16 N^{2}}{C^{2}\left(\frac{\mathrm{i} \omega_{b}}{Q_{0} \omega}+\frac{\omega_{b}^{2}}{\omega^{2}}-1\right)}}}{2 \mathrm{i} \omega \rho c_{0} \sqrt{1-2 \mathrm{i} V}} p(0),
$$


so that, using $2 \rho c_{0} / \omega m_{0}=4 N / C \sqrt{\mu}$, we obtain the BM displacement ratio $D=\Delta / \Delta_{s}$, as a function of only the nondimensional parameters defined above:

$$
D=\frac{4 N \mathrm{i} \sqrt{1-2 \mathrm{i} V} \exp (\phi)}{C \sqrt{\mu}\left(\frac{\mathrm{i} \omega_{n}}{Q \omega}+\frac{\omega_{n}^{2}}{\omega^{2}}-1\right) \sqrt[4]{1+\frac{16 N^{2}}{C^{2}\left(\frac{\omega_{b}^{2}}{\omega^{2}}+\frac{\mathrm{i} \omega_{b}}{Q \omega}-1\right)}} \sqrt[4]{1+\frac{16 N^{2}}{C^{2}\left(\frac{\mathrm{i} \omega_{n}}{Q \omega}+\frac{\omega_{n}^{2}}{\omega^{2}}-1\right)}}} .
$$

\section{Parametric variation with nondimensional parameters}

In this section we show how the amplitude and the phase of the normalized BM motion $D$, as calculated from Eq. (22), depends on the nondimensional parameters introduced in Section $2(Q, N, C$ and $V)$, so that we can clarify their physical significance. In Fig. 5 the amplitude and the phase of $D$ are plotted, as a function of the normalized angular frequency $\omega / \omega_{n}$, for different values of the nondimensional compressibility parameter, $C$, calculated at $\omega / \omega_{n}=1$, at which frequency $C$ is equal to $C_{n}$.
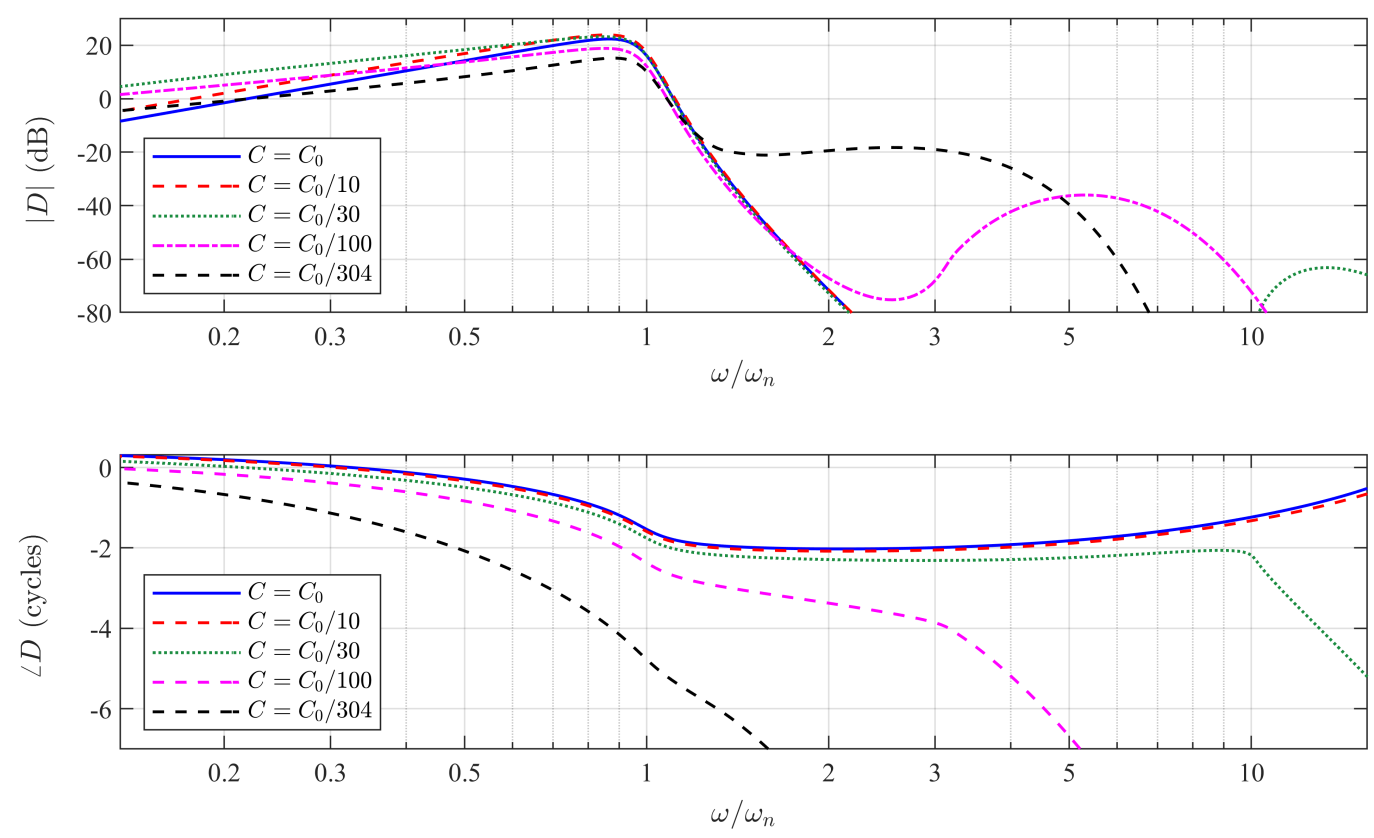

Figure 5: Variation of the amplitude (top) and of the phase (bottom) of the normalized BM displacement, for different values of the nondimensional compressibility parameter $C$, calculated at $\omega / \omega_{n}=1$, as a function of normalized excitation frequency. 
As $C$ is increased above the nominal value $C_{0}$, but less than $30 C_{0}$, the amplitude of the displacement ratio increases in the lower pass band. For values of $C$ greater than $100 C_{0}$, which will be seen to be relevant to the case of the rainbow sensor, the value of $D$ decreases at the characteristic frequency. In the stop band, the response decays rapidly, due to the high losses attributed to the imaginary part of the wavenumber there, as seen in Fig. 2a. For larger values of $C$, a second peak appears in the second pass band, as the wave again starts to propagate. In the phase plot of Fig. 5, it can be seen that the phase decreases gradually up to the peak frequency. In the stop band the phase shift is lower. In the second pass band, the phase again decreases due to forward wave propagation.

In Fig. 6, the amplitude and the phase of $D$ are plotted for different values of the nondimensional viscosity parameter, $V$, calculated at $\omega / \omega_{n}=1$. The nondimensional viscosity term has an effect only for very high values $\left(V>10^{4} V_{0}\right)$, for which the magnitude of $D$ is reduced, resulting in a lower peak around $\omega / \omega_{n}$.
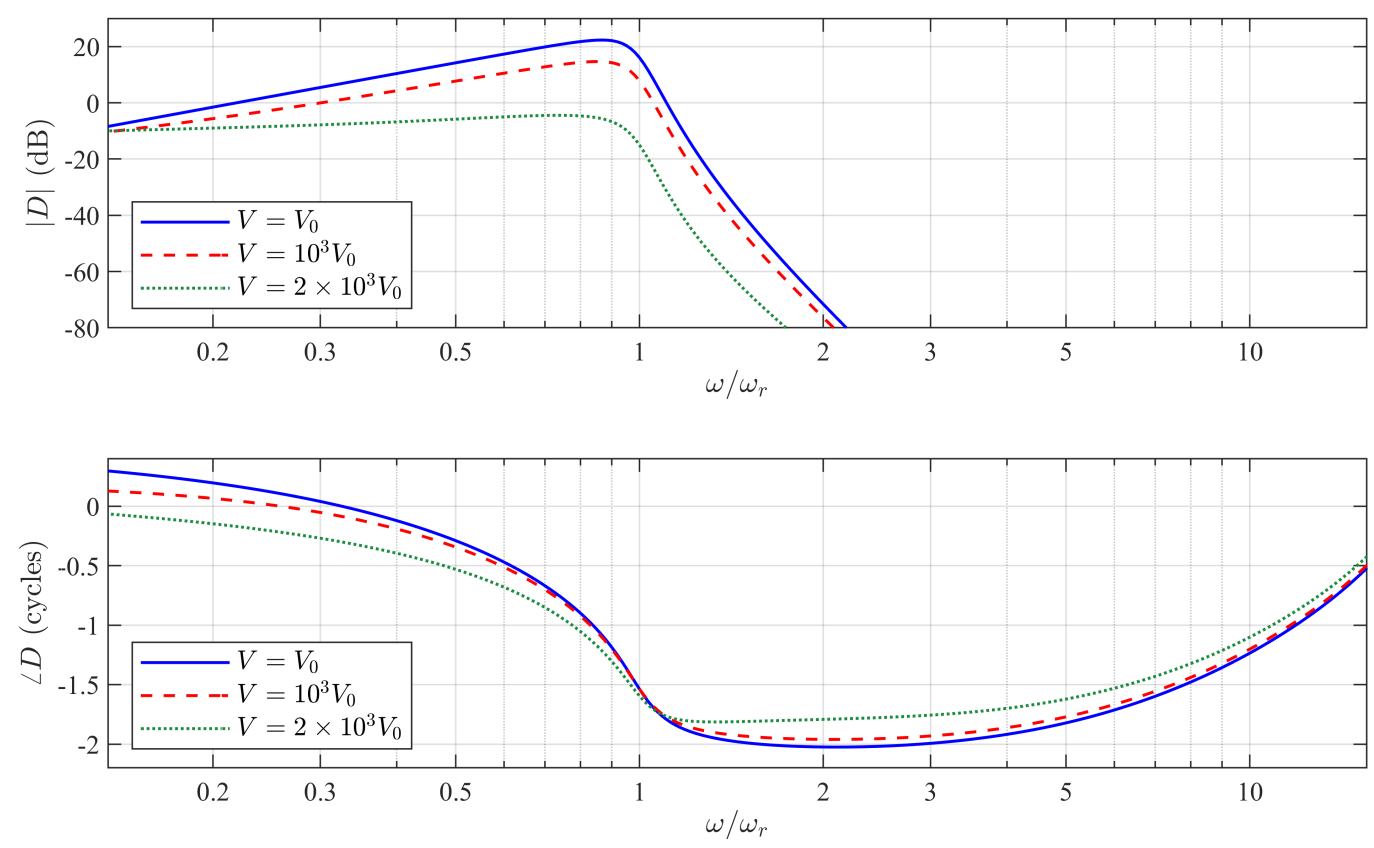

Figure 6: Variation of the amplitude (top) and of the phase (bottom) of the normalized BM displacement, for different values of the nondimensional viscosity parameter $V$, calculated at $\omega / \omega_{n}=1$, as a function of normalized excitation frequency. 
This is related to the increase of the real and imaginary part of the wavenumber, for high values of $V$, as seen in Fig. 2b. In particular, the high value of the imaginary part at lower frequencies greatly attenuates the wave, thus decreasing the value of $D$ and flattening the curve. This supports a previous study [30], in which it is shown that the effect of viscosity is important only at the apex of the cochlea, where the viscous boundary layer thickness becomes comparable to the height of the cochlea. It is also in agreement with experimental results in which it has been shown that an increase in fluid viscosity led to a decrease in the BM amplitude [31] and in the flattening of the response curve [32].

In Fig. 7 the amplitude and the phase of $D$ are plotted for different values of the quality factor, $Q$. The amplitude response becomes sharper around the natural frequency and shifts to higher frequencies with increasing $Q$. For $\omega / \omega_{n}>1$ the response decay becomes steeper as $Q$ is increased, while for $\omega / \omega_{n} \ll 1$ the amplitude is relatively unaffected. The phase response becomes steeper around the characteristic frequency with increasing $Q$. This corresponds to the increase of the real part of the wavenumber around $\omega / \omega_{n}=$ 1. 

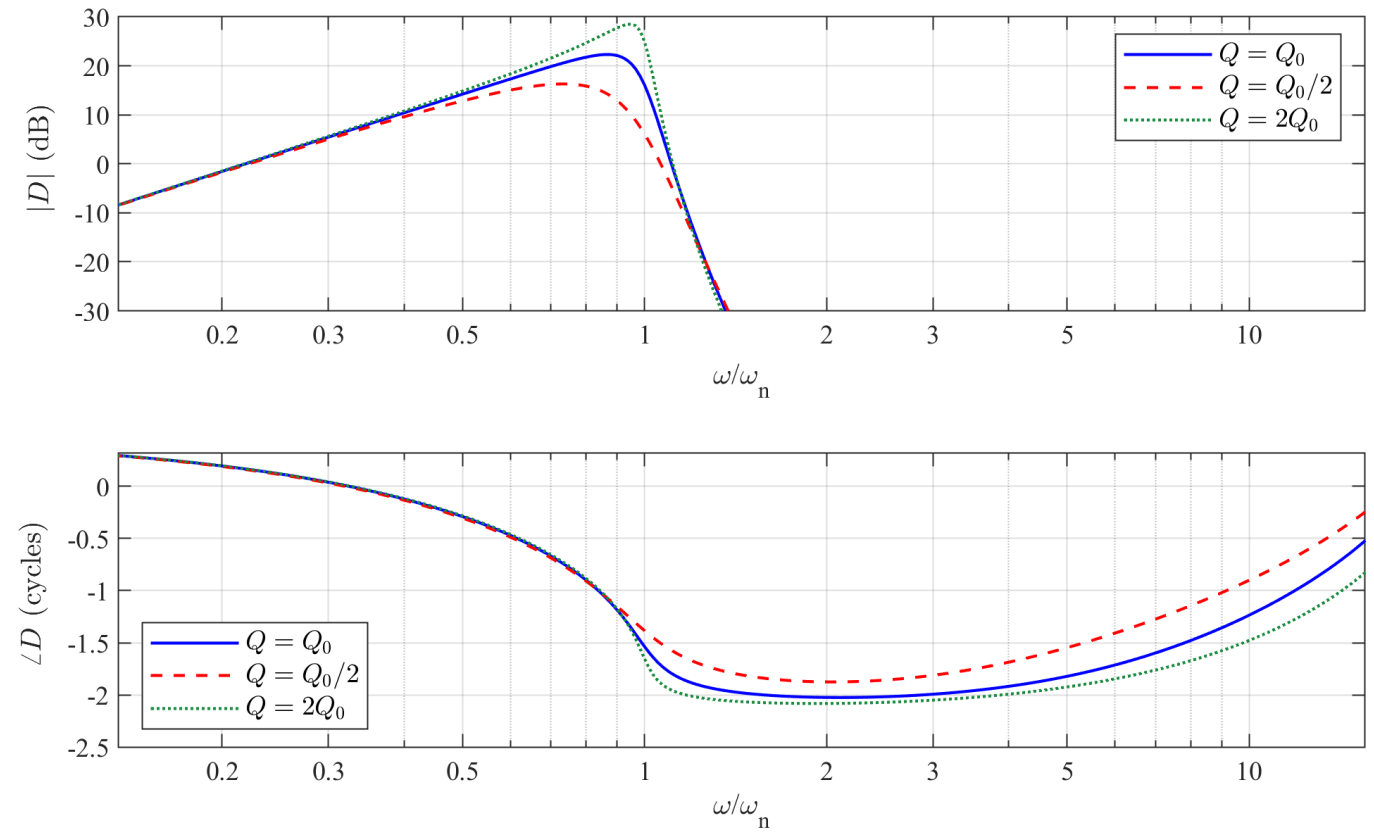

Figure 7: Variation of the amplitude (top) and of the phase (bottom) of the normalized $\mathrm{BM}$ displacement, for different values of the nondimensional quality factor $Q$, as a function of normalized excitation frequency. 
In Fig. 8 the amplitude and the phase of $D$ are plotted for different values of the nondimensional phase-shift parameter $N$. For low values of $N$, the response of the $\mathrm{BM}$ is almost symmetric on a log frequency axis, indicating weak fluid coupling and a phase shift of about half a cycle only occurs close to the characteristic frequency, implying a local resonance and no wave propagation. With increasing values of $N$, the phase change is larger and the amplitude response becomes asymmetrical, with a characteristic peak at sightly less than $\omega / \omega_{n}=1$, with a steep decrease after this, indicating strong fluid coupling. This is related to the dependence of the wavenumber on $N$, as shown in Fig. 2d. For small values of $N$, the real part of the wavenumber is small so that the wave speed is high and the pressure becomes almost uniform along the cochlea, driving the BM as a resonant second order system. The phase shift of $D$ is then only $\pi / 2$ at the BM resonance, as associated with a isolated resonator. These results are in accordance with [33], where the relationship between the BM mass and the coupled response is discussed. The more complete analysis here illustrates the dependence of the form of the coupled response not just on the BM mass via $\mu$, but on the more complicated nondimensional parameter, $N$.

In all the cases discussed above, the phase shift of the BM displacement ratio, given by Eq. (22), increases somewhat for frequencies much higher than the characteristic frequency. This is in contrast to what has been observed experimentally, as in [34]. However, this phase increase only occurs when the normalized magnitude response is lower than $-60 \mathrm{~dB}$, so that it does not affect the overall response in practice. 

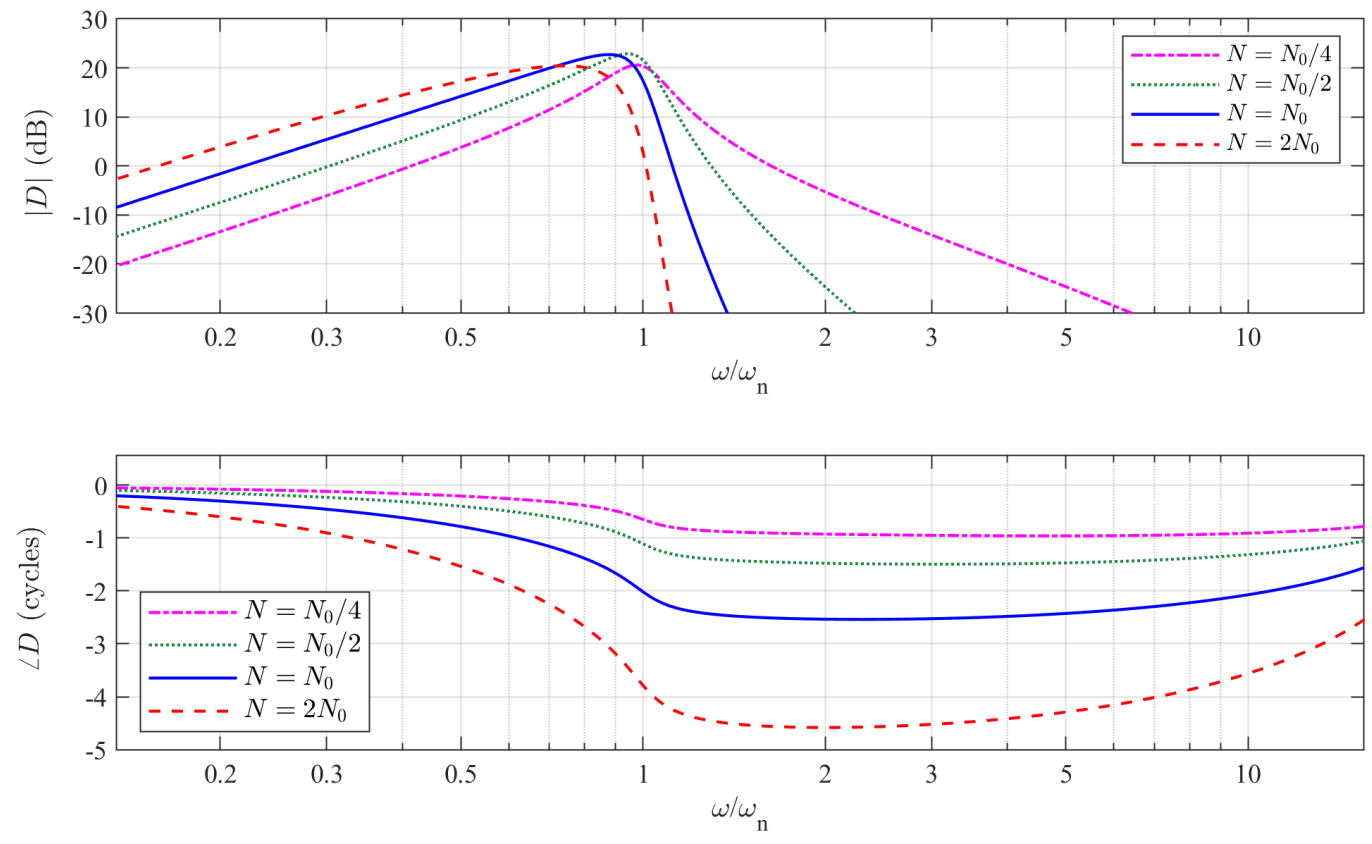

Figure 8: Variation of the amplitude (top) and of the phase (bottom) of the normalized BM displacement, for different values of the nondimensional parameter $N$, as a function of normalized excitation frequency. 


\subsection{Incompressible and inviscid case}

The contributions of compressibility and viscosity of the fluid to the displacement ratio can be neglected if $V$ and $C$ are much smaller than unity. This is valid for nominal physiological values of these parameters, as seen in Table 2. In this case, the wavenumber in Eq. (11) simplifies so that:

$$
k l=4 N \sqrt{\frac{\omega^{2}}{\omega_{n}^{2}+\mathrm{i} \omega_{n} \omega / Q-\omega^{2}}}
$$

i.e. the wavenumber is directly proportional to $N$ as seen in Figure 2d. Equation (22) for the displacement ratio then reduces to:

$$
D=\frac{4 N \mathrm{i} \exp \left\{-\mathrm{i} 4 N\left(\arcsin \left(\omega / \omega_{n}-\mathrm{i} /(2 Q)\right)-\arcsin \left(\omega / \omega_{b}-\mathrm{i} /(2 Q)\right)\right\}\right.}{C \sqrt{\mu}\left(\frac{\mathrm{i} \omega_{n}}{Q \omega}+\frac{\omega_{n}^{2}}{\omega^{2}}-1\right) \frac{4 N}{C} \sqrt[4]{\frac{1}{\left(\frac{\omega_{b}^{2}}{\omega^{2}}+\frac{\mathrm{i} \omega_{b}}{Q \omega}-1\right)}} \sqrt[4]{\frac{1}{\left(\frac{\omega_{n}^{2}}{\omega^{2}}+\frac{\mathrm{i} \omega_{n}}{Q \omega}-1\right)}}}
$$

which becomes, taking into account the relation $N=l /(4 h \mu)$ and making the approximation $\omega \ll \omega_{b}$ :

$$
D_{\text {incomp }}=\frac{\mathrm{i} \omega \sqrt{\omega_{b}} \exp \left\{-\mathrm{i} 4 N\left(\arcsin \left(\omega / \omega_{n}-\mathrm{i} /(2 Q)\right)\right\}\right.}{\sqrt{\mu}\left(\omega_{n}^{2}-\omega^{2}+\mathrm{i} \frac{\omega \omega_{n}}{Q}\right)^{3 / 4}} .
$$

Apart from an additional factor of $h$ and the absence of a factor of $\omega_{n}^{3 / 2}$, which give the correct nondimensionality, this expression is equivalent to that derived in [17] for the case of an incompressible and inviscid fluid.

The only direct effect of the mass ratio, $\mu$, on $D$ is to scale the overall response, whose shape is determined by $N$ and $Q$. Although the nondimensional parameter $N$ is seen to be a function of $\mu$ in the definition in Eq. 14c, its additional dependence on $l$ and $h$ means that in numerical simulations it can be varied while keeping $\mu$ constant, as in Fig. 4.13 of [35]. 


\section{Cochlea-inspired acoustic rainbow sensor}

Designs of acoustic rainbow sensor whose characteristics are inspired by the cochlea have been presented by several authors $[11,12,36]$. In particular, [36] investigated a design that aimed to achieve a smooth frequency response, as well as a similar spatial spectral analysis to that observed in the cochlea. Fig. 9a shows a schematic of this rainbow sensor, consisting of a main duct of constant cross section with an array of Helmholtz resonator side branches of varying dimensions. An element of the system consists of a segment of the main duct and a Helmholtz resonator side branch, whose lumped parameter approximation is shown in Fig. 9b. The series impedance, $Z_{1}$, is determined by the inertance of the fluid in the duct, $L_{D}$, which is assumed to be air

in this case, and the shunt impedance, $Z_{2}$, is the parallel combination of the compliance of the air in the duct, $C_{f}$, and the input impedance of the Helmholtz resonator, consisting of the compliance of the volume, $C_{H}$, the inertance of the neck, $L_{H}$, and the resistance of the neck, $R_{H}$. It was shown in [36] that a sufficient number of at least six elements per wavelength has to be used to achieve a smooth frequency response. Under these conditions, the size of the elements is small compared with the wavelength, justifying the use of the lumped parameter approximation, and the behavior of the array of elements can also be reasonably approximated by a continuous system, having smoothly varying properties, which can be analyzed using transmission line theory. The response of an example rainbow sensor taken from [36] is presented here, and is compared with the response of the cochlear model above. Results from these simulations illustrate the similarities of the two models.

\subsection{Wavenumber in the acoustic rainbow sensor}

Each element of the lumped parameter model of the system has a finite length, $l_{D}$, rather than having acoustic properties per unit length as in formal transmission line theory [37]. Therefore, the series, $Z_{1}$, and shunt, $Z_{2}$, acoustic impedances of an element, as shown in Fig. 9b, have to be normalized by $l_{D}$. An equivalent wavenumber for the transmission line can then be calculated as

$$
k=\frac{1}{\mathrm{i} l_{D}} \sqrt{\frac{Z_{1}}{Z_{2}}} .
$$




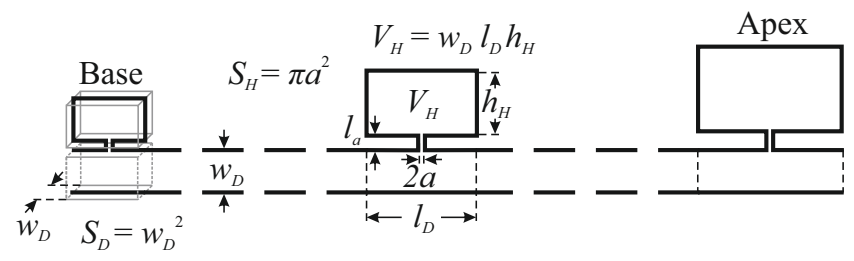

(a)

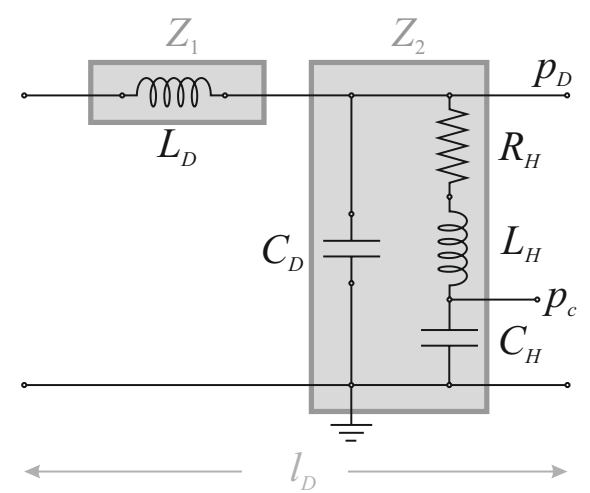

(b)

Figure 9: (a) Schematic of an acoustic rainbow sensor consisting of a square main duct of constant cross section $S_{D}=w_{D}^{2}$ and a number of Helmholtz resonator side branches. Also indicated are the length of an element of the system, $l_{D}$, the radius, $a$, and the length, $l_{a}$, of the resonator neck and the height, $h_{H}$, of the resonator cavity. Formulas for the area of the neck, $S_{H}$, and the volume of the cavity, $V_{H}$, are also given. The input end of the system is termed the 'base' and the other end is termed 'the apex', by analogy with the cochlea. (b) Equivalent circuit drawing of a lumped parameter model of an element of the transmission line, including the acoustic inertance, $L_{D}$, and acoustic compliance, $C_{D}$, of the duct, and the acoustic resistance, $R_{H}$, inertance, $L_{H}$, and compliance, $C_{H}$, of the Helmholtz resonator. The series impedance, $Z_{1}$, of the transmission line element is due to the duct inertance, and the shunt impedance is the parallel combination of the duct compliance and the RLC branch of the Helmholtz resonator impedance. Also noted are the acoustic pressure in the duct, $p_{D}$, which is analogous to the fluid pressure difference in the cochlea, and the pressure in the resonator cavity, $p_{c}$, which is analogous to the BM displacement in the cochlea. 
Using the explicit formulas of the series and shunt impedances, the spatially varying wavenumber of the transmission line can be written as [36]

$$
k=k_{0}\left[\frac{G^{2} \omega_{n}^{2}+\mathrm{i} \frac{\omega \omega_{n}}{Q}-\omega^{2}}{\omega_{n}^{2}+\mathrm{i} \frac{\omega \omega_{n}}{Q}-\omega^{2}}\right]^{1 / 2},
$$

where $k_{0}$ is $\omega / c_{0}$ and $c_{0}$ is the speed of sound in the fluid, which is air in this case, $\omega_{n}$ and $Q$ are the resonance angular frequency and the quality factor of a given element and $G$ is the bandgap ratio, in this case given by:

$$
G=\sqrt{1+H}
$$

in which

$$
H=\frac{C_{H}}{C_{D}}=\frac{V_{H}}{V_{D}}
$$

is the ratio of compliances, and hence the volumes, of the resonator cavity and the duct element. It can thus be seen that the limiting expression for the wavenumber in the acoustic rainbow sensor, when the elements are small compared with the wavelength, is of exactly the same form as that of the cochlear model considered in Section 2, Eq. (11). In the continuous limit, where the element length is infinitesimal, transmission line theory applies exactly, so that the pressure in the duct of the rainbow sensor is given by the well-known wave equation of Eq. (5), as the pressure in the cochlea. Furthermore, the bandgap ratio of the Helmholtz resonator here, $\sqrt{1+H}$, is analogous to the term $\sqrt{1+\frac{16 N^{2}}{C_{n}^{2}}}$ in Eq. (13). Hence, the effective value of the nondimensional compressibility parameter at resonance defined in Section 2 for the acoustic rainbow sensor is equal to:

$$
C_{n}=\frac{4 N}{\sqrt{H}}
$$

which is about 9 for the design of sensor described in [36]. The corresponding normalized compressibility parameter $C$, which is equal to $C=C_{n} \omega / \omega_{n}$, is thus significantly larger for this acoustic rainbow sensor than it is in the cochlea, and hence plays a greater role in determining the response. In fact the nondimensional compressibility factor for this rainbow sensor is about 304 times that in the cochlear model, as given in Table 2, which is why 
this value was chosen in Fig. 5. Apart from this, the analogy between the rainbow sensor and the cochlea can be further explored by considering the lumped parameter approximation to an element in the passive cochlea, which is exactly as in Fig. 9b, except that the inertance of the fluid in the sensor duct is replaced by the inertance of the fluid in the cochlear chambers, and the Helmholtz resonator is replaced by the mass-spring-damper model of the BM. The shunt compliance term is not normally included in lumped parameter models of the cochlea [17], since it corresponds to the compressibility of the fluid in the cochlear chambers, which is very small, as seen above. The pressure in the rainbow sensor duct, $p_{D}$ in Fig. $9 \mathrm{~b}$, is thus analogous to the pressure difference across the BM in the cochlea [17] and the pressure in the volume of the Helmholtz resonator, $p_{c}$, in Fig. 9b, is analogous to the volume velocity through the RLC circuit representing the mass-springdamper model of the BM, multiplied by the impedance of the spring. The resonator pressure, $p_{c}$, is thus analogous to the displacement of the BM in the cochlea, scaled by a factor of $C_{H}$.

\subsection{Response of an example acoustic rainbow sensor}

Table 3 shows the dimensions of an example rainbow sensor taken from [36], which has 50 elements and whose main characteristics are presented below. The variable $l_{H}$ in Table 3 represents the effective length of the Helmholtz resonator neck, which is larger than the physical length of the resonator neck since it takes into account the end corrections due to sound radiation from the two ends.

As in the cochlea with high values of $C_{0}$, the rainbow sensor wavenumber is characterized by a low frequency pass band, a stop band, and a high frequency pass band. It should be noted that the bandgap in the rainbow sensor is constant for all the elements, since $H$, in Eq. (29), is designed to be equal to 1 throughout the sensor, which is achieved by varying the length of the duct between resonators.

The modulus and phase of the rainbow sensor's frequency response are plotted in Fig. 10, in terms of the acoustic pressure in the cavities, corresponding to the BM displacement in the cochlea, for three different elements along the rainbow sensor. The response of the rainbow sensor is calculated with a distributed Transfer Matrix method, which accounts for wave propagation in the ducts and cavities of the system and interference effects between the discrete elements, as in [36], in contrast to the use of the WKB method employed to give the results for the analogous plot for the cochlea in Fig. 6. 
Table 3: Design parameter values of the first, $25^{t h}$ and $50^{t h}$ element of the example acoustic rainbow sensor. The neck radius is $a=0.5 \mathrm{~mm}$ and the duct width is $w_{D}=7.3 \mathrm{~mm}$ for all the elements.

\begin{tabular}{ccccccc}
\hline$n$ & $f_{1}(\mathrm{~Hz})$ & $Q_{1}$ & $l_{D}(\mathrm{~mm})$ & $h_{H}(\mathrm{~mm})$ & $l_{a}(\mathrm{~mm})$ & $l_{H}(\mathrm{~mm})$ \\
\hline 1 & 3400 & 17.5 & 3.8 & 7.3 & 0.31 & 1 \\
25 & 1035 & 9.7 & 16.9 & 7.3 & 1.7 & 2.4 \\
50 & 300 & 5.2 & 79.8 & 7.3 & 5.42 & 6.2 \\
\hline
\end{tabular}

The similarities between the two cases can be seen by comparing the response of the rainbow sensor at $n=12$, given in Fig. 10, and that of the cochlea, with high fluid compressibility, given in Fig. 5, which for $C=304 C_{0}$, gives a bandgap ratio of $\sqrt{2}$. As in Fig. 5 , the rainbow sensor response in Fig. 10 increases in the lower pass band up to a frequency lower than the natural frequency. In the stop band region the response decreases rapidly. In the upper band, the response first peaks and then continues to decrease, albeit more gradually. Internal resonances within the individual element enhance the higher frequency peak in the discrete acoustic rainbow sensor compared with the continuous cochlear model [36]. At higher frequencies, the response of the rainbow sensor is dominated by interfering acoustic phenomena within the elements, which lead to additional peaks.

The phase responses are also similar between the two figures: there is a gradual decrease up to the peak frequency and a lower phase shift in the stop band. The inversion of the phase observed in the basal element is due to interfering phenomena at higher frequencies. In the second pass band, the phase again decreases due to forward wave propagation. 

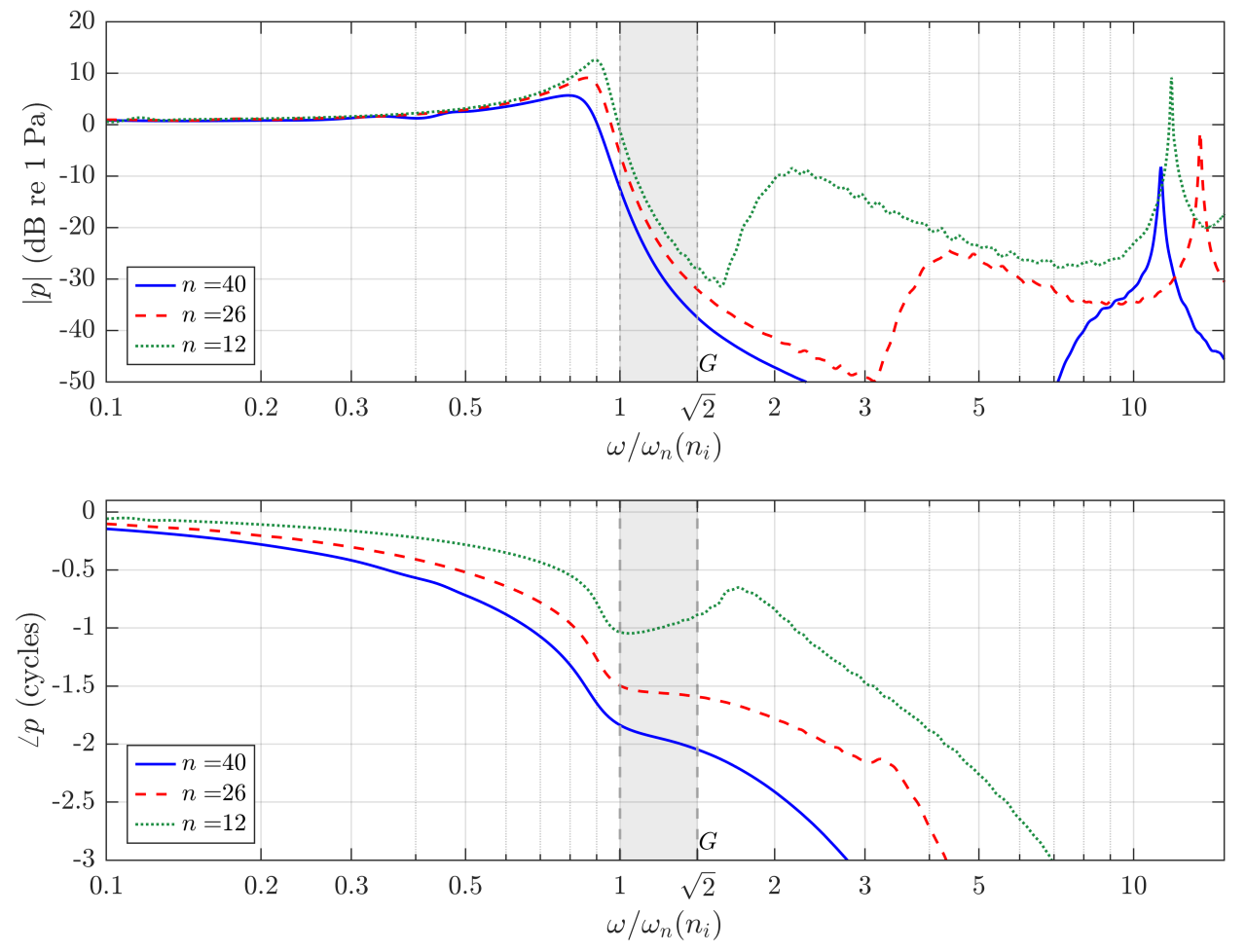

Figure 10: Acoustic pressure in the resonator cavities plotted against normalized frequency for three different resonators, modulus (upper), and phase (lower). The dashed vertical lines define the limits of the stop band, which is shaded in light grey. 


\section{Conclusions}

An analytic WKB solution for the wave equation in the passive cochlea has been derived, including the effects of both viscosity and compressibility in the cochlear fluids, which reduces to an earlier result [17], if the effects of viscosity or compressibility are ignored. This allows the response of the cochlea to be expressed in terms of four nondimensional parameters, two dependent on the viscosity and compressibility of the fluid, one dependent on the damping of the basilar membrane, and finally a previously derived nondimensional phase-shift parameter [17]. For the parameters found in the normal cochlea, however, the effect of the fluid viscosity and compressibility is very small. A physical interpretation can then be put on the behavior of the wave by considering the parametric variation of the cochlear response with each of these parameters. It is found that the nondimensional phase-shift parameter determines not only the high frequency phase shift [17] but also the nature of the interaction between the structural dynamics of the BM and the fluid inertia. If this parameter is large, the fluid-structural coupling is strong and a propagating wave is generated for frequencies up to about the natural frequency, with strong attenuation above this, giving a very asymmetrical frequency response. If this parameter is small, the fluid-structural coupling is weak, and the elements of the basilar membrane respond independently, driven by the constant pressure in the fluid, generating an almost symmetrical frequency response function when plotted on a log scale. Although the phaseshift parameter that determines the shape of the response is proportional to the square root of the ratio of the mass per unit length of the fluid in the chambers to the mass per unit length of the BM [33], it also depends on the ratio of the characteristic length of the exponential decay in frequency along the cochlea to the effective height of the fluid chambers. Therefore, the mass ratio by itself only changes the amplitude and not the form of the response

if the phase-shift parameter is kept constant and the characteristic length of the cochlea is varied accordingly.

Some features of the cochlear response with values of the compressibility parameter above that normally encountered in the cochlea turn out to be relevant to the behavior of acoustic rainbow sensors. It is shown that the limiting expression for the wavenumber in the case of an acoustic rainbow sensor with a smoothly varying frequency response takes the same form as that in the cochlea with fluid compressibility. A second pass band, which cuts in above the frequency of the stop band, is seen to be important in 
determining the response of the acoustic rainbow sensor and the cochlea with high fluid compressibility. This explains the second peak observed in the predicted cochlear response when it has high fluid compressibility. Although in principle this peak is also present in the normal cochlea, it would occur at a sufficiently high frequency for it to be negligible.

\section{Acknowledgements}

Riccardo Marrocchio is supported by a studentship from the UK Engineering and Physical Sciences Research Council (EPSRC), associated with the DigiTwin project (EP/R006768/1). Angelis Karlos is supported by the National Science Centre in Poland (2018/31/B/ST8/00753).

\section{Appendix. Solution of the phase integral}

The integral in Eq. (18) can be written, using Eq. (10) and Eq. (9), as:

$$
\phi=-\mathrm{i} b \frac{w}{c_{0}} \int_{0}^{x} \sqrt{\frac{a c_{0}^{2}}{\left(\omega_{b} \exp \left(-x^{\prime} / l\right)+i \omega / Q\right)^{2}-\omega^{2}}+1} \mathrm{~d} x^{\prime}
$$

where we defined:

$$
\begin{aligned}
& a=\frac{2 \rho}{h m_{0}}, \\
& b=\sqrt{1-\frac{2 i \nu}{h^{2} \omega \rho}},
\end{aligned}
$$

and at the denominator under the square root of the integrand in Eq. (A.1) we completed the square and assume that $1 / Q^{2}$ is small compared with 1 , as in [17]. We now change variable by using $\omega_{n}=\omega_{b} \exp \left(-x^{\prime} / l\right)$ and obtain:

$$
\phi=\mathrm{i} \frac{l b \omega}{c_{0}} \int_{w_{b}}^{w_{n}} \frac{1}{\omega_{n}} \sqrt{\frac{a c_{0}^{2}}{\left(\omega_{n}+\mathrm{i} \omega / Q\right)^{2}-\omega^{2}}+1} \mathrm{~d} \omega_{n} .
$$

By defining the new variable $t=\left(\omega_{n}+\frac{i \omega}{Q}\right)$ we have:

$$
\phi=\mathrm{i} \frac{l b \omega}{c_{0}} \int_{\left(\mathrm{i} \omega / Q+\omega_{b}\right)}^{\left(\mathrm{i} \omega / Q+\omega_{n}\right)} \frac{1}{(t-\mathrm{i} \omega / Q)} \frac{\sqrt{a c_{0}^{2}+t^{2}-\omega^{2}}}{\sqrt{t^{2}-\omega^{2}}} \mathrm{~d} t .
$$


Taking into account that:

$$
\frac{1}{t-\mathrm{i} \omega / Q}=\frac{\mathrm{i} \omega}{Q\left(t^{2}+\omega^{2} / Q^{2}\right)}+\frac{t}{t^{2}+\omega^{2} / Q^{2}},
$$

the integral becomes:

$$
\begin{aligned}
& \phi=\mathrm{i} \frac{l b \omega}{c_{0}}\left[\int_{\left(\mathrm{i} \omega / Q+\omega_{b}\right)}^{\left(\mathrm{i} \omega / Q+\omega_{n}\right)} \frac{i \omega \sqrt{a c_{0}^{2}+t^{2}-\omega^{2}}}{Q\left(t^{2}+\omega^{2} / Q^{2}\right) \sqrt{t^{2}-\omega^{2}}} \mathrm{~d} t\right. \\
& \left.+\int_{\left(\mathrm{i} \omega / Q+\omega_{b}\right)}^{\left(\mathrm{i} \omega / Q+\omega_{n}\right)} \frac{t}{t^{2}+\omega^{2} / Q^{2}} \frac{\sqrt{a c_{0}^{2}+t^{2}-\omega^{2}}}{\sqrt{t^{2}-\omega^{2}}} \mathrm{~d} t\right] .
\end{aligned}
$$

Now we consider each integral in the square bracket in turn. We call them respectively $I_{1}$ and $I_{2}$. Starting from the last one, if we change variable using the substitution $s=t^{2}$ we obtain:

$$
I_{2}=\int_{\left(\mathrm{i} \omega / Q+\omega_{b}\right)^{2}}^{\left(\mathrm{i} \omega / Q+\omega_{n}\right)^{2}} \frac{1}{2 s+2 \omega^{2} / Q^{2}} \frac{\sqrt{a c_{0}^{2}+s-\omega^{2}}}{\sqrt{s-\omega^{2}}} \mathrm{~d} s .
$$

The integrand can be decomposed as:

$$
\begin{aligned}
& I_{2}=1 / 2 \int_{\left(\mathrm{i} \omega / Q+\omega_{b}\right)^{2}}^{\left(\mathrm{i} \omega / Q+\omega_{n}\right)^{2}} \frac{1}{\sqrt{s-\omega^{2}} \sqrt{a c_{0}^{2}+s-w^{2}}} \mathrm{~d} s \\
& +1 / 2 \int_{\left(\mathrm{i} \omega / Q+\omega_{b}\right)^{2}}^{\left(\mathrm{i} \omega / Q+\omega_{n}\right)^{2}} \frac{4 a c_{0}^{2}-\left(4+4 / Q^{2}\right) \omega^{2}}{\sqrt{s-\omega^{2}} \sqrt{a c_{0}^{2}+s-w^{2}}\left(4 s+4 \omega^{2} / Q^{2}\right)} \mathrm{d} s,
\end{aligned}
$$

which we call, respectively, $I_{2 A}$ and $I_{2 B}$. If we define $q=\sqrt{s-\omega^{2}}$ then:

$$
I_{2 A}=\int_{\sqrt{\left(\mathrm{i} \omega / Q+\omega_{b}\right)^{2}-\omega^{2}}}^{\sqrt{\left(\mathrm{i} \omega / Q+\omega_{n}\right)^{2}-\omega^{2}}} \frac{1}{\sqrt{a c_{0}^{2}+q^{2}}} \mathrm{~d} q,
$$

and by using $r=q / \sqrt{a c_{0}^{2}+q^{2}}$ :

$$
I_{2 A}=\int_{\sqrt{\left(\mathrm{i} \omega / Q+\omega_{b}\right)^{2}-\omega^{2}} / \sqrt{a c_{0}^{2}-\omega^{2}+\left(\mathrm{i} \omega / Q+\omega_{b}\right)^{2}}}^{\sqrt{\left(\mathrm{i} \omega / Q+\omega_{n}\right)^{2}-\omega^{2}} / \sqrt{a c_{0}^{2}-\omega^{2}+\left(\mathrm{i} \omega / Q+\omega_{n}\right)^{2}}} \frac{1}{1-r^{2}} \mathrm{~d} r
$$

so that:

$$
\begin{aligned}
& I_{2 A}=\operatorname{arctanh}\left(\frac{\sqrt{\left(\mathrm{i} \omega / Q+\omega_{n}\right)^{2}-\omega^{2}}}{\sqrt{a c_{0}^{2}-\omega^{2}+\left(\mathrm{i} \omega / Q+\omega_{n}\right)^{2}}}\right) \\
& -\operatorname{arctanh}\left(\frac{\sqrt{\left(\mathrm{i} \omega / Q+\omega_{b}\right)^{2}-\omega^{2}}}{\sqrt{a c_{0}^{2}-\omega^{2}+\left(\mathrm{i} \omega / Q+\omega_{b}\right)^{2}}}\right) .
\end{aligned}
$$


Now we go back to the integral $I_{2 B}$ in Eq. (A.8) for which we use the substitution $m=\sqrt{s-\omega^{2}} / \sqrt{a c_{0}^{2}+s-\omega^{2}}$ so that:

$$
I_{2 B}=\int_{\alpha}^{\beta} \frac{4 a c_{0}^{2}-\left(4+4 / Q^{2}\right) \omega^{2}}{4 \omega^{2}+4 / Q^{2} \omega^{2}-m^{2}\left(4 / Q^{2} \omega^{2}-4\left(a c_{0}^{2}-\omega^{2}\right)\right)} \mathrm{d} d m
$$

where $\alpha=\sqrt{\left(\mathrm{i} \omega / Q+\omega_{b}\right)^{2}-\omega^{2}} / \sqrt{a c_{0}^{2}-\omega^{2}+\left(\mathrm{i} \omega / Q+\omega_{b}\right)^{2}}$ and $\beta=\sqrt{\left(\mathrm{i} \omega / Q+\omega_{b}\right)^{2}-\omega^{2}} / \sqrt{\left.a c_{0}^{2}-\omega^{2}+\mathrm{i} i \omega / Q+\omega_{b}\right)^{2}}$. The result of this integral is:

$$
\begin{aligned}
& I_{2 B}=\frac{\sqrt{4 a c_{0}^{2}-\left(4+4 / Q^{2}\right) \omega^{2}}}{\omega \sqrt{4+4 / Q^{2}}}\left[\operatorname{arctanh}\left(\frac{\sqrt{4 a c_{0}^{2}-\left(4+4 / Q^{2}\right) \omega^{2}}}{\omega \sqrt{4+4 / Q^{2}}} \alpha\right)\right. \\
& \left.-\operatorname{arctanh}\left(\frac{\sqrt{4 a c_{0}^{2}-\left(4+4 / Q^{2}\right) \omega^{2}}}{\omega \sqrt{4+4 / Q^{2}}} \beta\right)\right] .
\end{aligned}
$$

Now going back to $I_{1}$ in Eq. (A.6), we can rewrite this as:

$$
\begin{aligned}
& I_{1}=\frac{Q}{4 \omega \sqrt{a c_{0}^{2}-\omega^{2}}}\left[\int_{\left(\mathrm{i} \omega / Q+\omega_{b}\right)}^{\left(\mathrm{i} \omega / Q+\omega_{n}\right)} \frac{4 \omega^{2} \sqrt{-a c_{0}^{2}+\omega^{2}}}{Q^{2} \sqrt{-t^{2}+\omega^{2}} \sqrt{-a c_{0}^{2}-t^{2}+\omega^{2}}} \mathrm{~d} t\right. \\
& \left.+\int_{\left(\mathrm{i} \omega / Q+\omega_{b}\right)}^{\left(\mathrm{i} \omega / Q+\omega_{n}\right)} \frac{4 \omega^{2} \sqrt{-a c_{0}^{2}+\omega^{2}}\left(4 a c_{0}^{2}-\left(4+4 / Q^{2}\right) \omega^{2}\right)}{Q^{2} \sqrt{-t^{2}+\omega^{2}} \sqrt{-a c_{0}^{2}-t^{2}+\omega^{2}}\left(4 t^{2}+4 \omega^{2} / Q^{2}\right)} \mathrm{d} t\right] .
\end{aligned}
$$

We call the integrals within the square brackets $I_{1 A}$ and $I_{1 B}$ respectively. Starting from the first one, if we define $\theta=-\arcsin (t / \omega)$, then we obtain:

$$
I_{1 A}=\frac{4 \omega^{2}}{Q^{2}} \int_{-\arcsin \left(\mathrm{i} / Q+\omega_{n} / \omega\right)}^{-\arcsin \left(\mathrm{i} / Q+\omega_{n} / \omega\right)} \frac{\mathrm{d} \theta}{\sqrt{1-\frac{\omega^{2}}{\omega^{2}-a c_{0}^{2}} \sin ^{2}(\theta)}}
$$

which is the difference of two elliptic integrals of the first kind, indicated as:

$$
\begin{aligned}
& I_{1 A}=\frac{4 \omega^{2}}{Q^{2}}\left[\mathrm{~F}\left(-\arcsin \left(\mathrm{i} / Q+\omega_{n} / \omega\right), \frac{\omega^{2}}{\omega^{2}-a c_{0}^{2}}\right)\right. \\
& \left.-\mathrm{F}\left(-\arcsin \left(\mathrm{i} / Q+\omega_{b} / \omega\right), \frac{\omega^{2}}{\omega^{2}-a c_{0}^{2}}\right)\right] .
\end{aligned}
$$


By using the same change of variable, the integral $I_{1 B}$ becomes:

$$
I_{1 B}=4\left(a c_{0}^{2}-\left(1+Q^{-2}\right) \omega^{2}\right) \int_{-\arcsin \left(\frac{i}{Q}+\frac{\omega_{b}}{\omega}\right)}^{-\arcsin \left(\frac{i}{Q}+\frac{\omega_{n}}{\omega}\right)} \frac{\mathrm{d} \theta}{\left(1+Q^{2} \sin ^{2}(\theta)\right) \sqrt{1-\frac{\omega^{2}}{\omega^{2}-a c_{0}^{2}} \sin ^{2}(\theta)}}
$$

which is the difference of two elliptic integrals of the third kind:

$$
\begin{aligned}
& I_{1 B}=\left(4 ( a c _ { 0 } ^ { 2 } - ( 1 + Q ^ { - 2 } ) \omega ^ { 2 } ) \left[\Pi\left(-Q^{2} ;-\arcsin \left(\mathrm{i} / Q+\omega_{n} / \omega\right), \frac{\omega^{2}}{\omega^{2}-a c_{0}^{2}}\right)\right.\right. \\
& \left.-\Pi\left(-Q^{2} ;-\arcsin \left(\mathrm{i} / Q+\omega_{b} / \omega\right), \frac{\omega^{2}}{\omega^{2}-a c_{0}^{2}}\right)\right] .
\end{aligned}
$$

Then, by putting together all the results above, the integral $\phi$ becomes:

$$
\begin{aligned}
& \phi=\frac{\mathrm{i} l b \omega}{c_{0}}\left[\frac{1}{Q \sqrt{-1+\frac{16 N^{2}}{C^{2}}}} \mathrm{~F}\left(-\arcsin \left(\mathrm{i} / Q+\omega_{n} / \omega\right), \frac{\omega^{2}}{\omega^{2}-a c_{0}^{2}}\right)\right. \\
& +\Pi\left(-Q^{2} ;-\arcsin \left(\mathrm{i} / Q+\omega_{n} / \omega\right), \frac{\omega^{2}}{\omega^{2}-a c_{0}^{2}}\right) \\
& +\operatorname{arctanh}\left(\frac{\sqrt{\left(\mathrm{i} \omega / Q+\omega_{n}\right)^{2}-\omega^{2}}}{\sqrt{a c_{0}^{2}-\omega^{2}+\left(\mathrm{i} \omega / Q+\omega_{n}\right)^{2}}}\right) \\
& +\frac{\sqrt{4 a c_{0}^{2}-\left(4+4 / Q^{2}\right) \omega^{2}}}{\omega \sqrt{4+4 / Q^{2}}}\left[\operatorname { a r c t a n } \left(\frac{\sqrt{4 a c_{0}^{2}-\left(4+4 / Q^{2}\right) \omega^{2}}}{\omega \sqrt{4+4 / Q^{2}}}\right.\right. \\
& \left.\left.\times \frac{\sqrt{\left(\mathrm{i} \omega / Q+\omega_{n}\right)^{2}-\omega^{2}}}{\sqrt{a c_{0}^{2}-\omega^{2}+\left(\mathrm{i} \omega / Q+\omega_{n}\right)^{2}}}\right)\right]+O\left(\frac{\omega}{\omega_{b}}\right),
\end{aligned}
$$

where $O\left(\omega / \omega_{b}\right)$ include the terms from the lower limit of integration. If we know use the definitions of the nondimensional variables introduced in 
Eq. (14), $\phi$ can be written as:

$$
\begin{aligned}
& \phi=\mathrm{i} C \sqrt{1-2 \mathrm{i} V}\left[\frac{1}{Q \sqrt{-1+\frac{16 N^{2}}{C^{2}}}} \mathrm{~F}\left(-\arcsin \left(\mathrm{i} / Q+\omega_{n} / \omega\right), \frac{1}{\sqrt{1-\frac{16 N^{2}}{C^{2}}}}\right)\right. \\
& +\frac{4 \omega^{2}}{Q} \sqrt{-1+\frac{16 N^{2}}{C^{2}}} \Pi\left(-Q^{2} ;-\arcsin \left(\mathrm{i} / Q+\omega_{n} / \omega\right), \frac{1}{\sqrt{1-\frac{16 N^{2}}{C^{2}}}}\right) \\
& +\operatorname{arctanh}\left(\frac{\sqrt{\left(\mathrm{i} / Q+\omega_{n} / \omega\right)^{2}-1}}{\sqrt{16 N^{2} / C^{2}-1+\left(\mathrm{i} / Q+\omega_{n} / \omega\right)^{2}}}\right) \\
& \left.+\sqrt{-1+\frac{16 N^{2}}{C^{2}}} \arctan \left(\frac{\sqrt{16 N^{2} / C^{2}-1} \sqrt{\left(\mathrm{i} / Q+\omega_{n} / \omega\right)^{2}-1}}{\sqrt{16 N^{2} / C^{2}-1+\left(\mathrm{i} / Q+\omega_{n} / \omega\right)^{2}}}\right)\right] \\
& +O\left(\frac{\omega}{\omega_{b}}\right) \text {, }
\end{aligned}
$$

where again we assume that $1-1 / Q^{2}$ is small compared with 1 . Finally, as we are interested in the form of the solution for excitation frequencies such that $\omega \ll \omega_{b}$, we can neglect the term of order $O\left(\omega / \omega_{b}\right)$ and we obtain the solution presented in Eq.(19).

\section{References}

[1] R. F. P. Dallos, A. Popper, The Cochlea: Springer Handbook of Auditory Research, Springer-Verlag, 1996.

[2] S. J. Elliott, G. Ni, B. R. Mace, B. Lineton, A wave finite element analysis of the passive cochlea, The Journal of the Acoustical Society of America 133 (3) (2013) 1535-1545. doi:10.1121/1.4790350.

[3] K. L. Tsakmakidis, A. D. Boardman, O. Hess, 'Trapped rainbow' storage of light in metamaterials, Sci Rep 450 (2007) 397-401. doi:https://doi.org/10.1038/nature06285.

[4] M. S. Jang, H. Atwater, Plasmonic rainbow trapping structures for light localization and spectrum splitting, Phys. Rev. Lett. 107 (2011) 207401. doi:10.1103/PhysRevLett.107.207401. 
[5] A. Colombi, D. Colquitt, P. Roux, S. Guenneau, R. V. Craster, A seismic metamaterial: The resonant metawedge, Sci Rep 6 (2016). doi:https://doi.org/10.1038/srep27717.

[6] Z. Tian, L. Yu, Rainbow trapping of ultrasonic guided waves in chirped phononic crystal plates, Sci Rep 7 (2017). doi:https://doi.org/10.1038/srep40004.

[7] E. A. Skelton, R. V. Craster, A. Colombi, D. J. Colquitt, The multiphysics metawedge: graded arrays on fluid-loaded elastic plates and the mechanical analogues of rainbow trapping and mode conversion, New Journal of Physics 20 (5) (2018) 053017. doi:10.1088/1367-2630/aabecf.

[8] H. Meng, D. Chronopoulos, A. Fabro, W. Elmadih, I. Maskery, Rainbow metamaterials for broadband multi-frequency vibration attenuation: Numerical analysis and experimental validation, Journal of Sound and Vibration 465 (2020) 115005. doi:https://doi.org/10.1016/j.jsv.2019.115005.

[9] G. J. Chaplain, J. M. De Ponti, G. Aguzzi, A. Colombi, R. V. Craster, Topological rainbow trapping for elastic energy harvesting in graded su-schrieffer-heeger systems, Phys. Rev. Applied 14 (2020) 054035. doi:10.1103/PhysRevApplied.14.054035.

[10] J. Zhu, Y. Chen, X. Zhu, F. J. Garcia-Vidal, X. Yin, W. Zhang, X. Zhang, Acoustic rainbow trapping, Scientific Reports 3 (1) (2013). doi:10.1038/srep01728.

[11] L. Zhao, S. Zhou, Compact acoustic rainbow trapping in a bioinspired spiral array of graded locally resonant metamaterials, Sensors (Basel) 19 (4) (2019). doi:10.3390/s19040788.

[12] X. Ni, Y. Wu, Z. G. Chen, L. Y. Zheng, Y. L. Xu, P. Nayar, X. P. Liu, M. H. Lu, Y. F. Chen, Acoustic rainbow trapping by coiling up space, Sci Rep 4 (2014) 7038. doi:10.1038/srep07038.

[13] M. Rupin, G. Lerosey, J. de Rosny, F. Lemoult, Mimicking the cochlea with an active acoustic metamaterial, New Journal of Physics 21 (9) (2019). doi:10.1088/1367-2630/ab3d8f. 
[14] N. Jimenez, V. Romero-Garcia, V. Pagneux, J. P. Groby, Rainbowtrapping absorbers: Broadband, perfect and asymmetric sound absorption by subwavelength panels for transmission problems, Sci Rep 7 (1) (2017) 13595. doi:10.1038/s41598-017-13706-4.

[15] F. Zangeneh-Nejad, R. Fleury, Active times for acoustic metamaterials, Reviews in Physics 4 (2019). doi:10.1016/j.revip.2019.100031.

[16] P. Deepu, Cochlear mechanics with fluid viscosity and compressibility, Physical Review E 99 (3) (2019) 032417. doi:10.1103/PhysRevE.99.032417.

[17] G. Zweig, R. Lipes, J. R. Pierce, The cochlear compromise, The Journal of the Acoustical Society of America 59 (4) (1976) 975-982. doi:10.1121/1.380956.

[18] C. R. Steele, C. E. Miller, An improved WKB calculation for a twodimensional cochlear model, The Journal of the Acoustical Society of America 68 (1) (1980) 147-148. doi:10.1121/1.384640.

[19] S. T. Neely, Finite difference solution of a two-dimensional mathematical model of the cochlea, The Journal of the Acoustical Society of America 69 (5) (1981) 1386-1393. doi:10.1121/1.385820.

[20] S. J. Elliott, G. Ni, An elemental approach to modelling the mechanics of the cochlea, Hearing Research 360 (2018) 14-24. doi:10.1016/j.heares.2017.10.013.

[21] L. A. Taber, C. R. Steele, Cochlear model including three-dimensional fluid and four modes of partition flexibility, The Journal of the Acoustical Society of America 70 (2) (1981) 426-436. doi:10.1121/1.386785.

[22] C. R. Steele, L. A. Taber, Comparison of WKB calculations and experimental results for three-dimensional cochlear models, The Journal of the Acoustical Society of America 65 (4) (1979) 1007-1018. doi:10.1121/1.382570.

[23] R. Mathews, R.; Walker, Mathematical Methods of Physics, Benjamin, New York, 1964. 
[24] E. de Boer, M. Viergever, Validity of the Liouville-Green (or WKB) method for cochlear mechanics, Hearing Research 8 (2) (1982) 131-155. doi:10.1016/0378-5955(82)90071-5.

[25] L. Watts, The mode-coupling Liouville-Green approximation for a twodimensional cochlear model, The Journal of the Acoustical Society of America 108 (5) (2000) 2266-2271. doi:10.1121/1.1310194.

[26] L. Robles, M. A. Ruggero, Mechanics of the Mammalian Cochlea, Physiological Reviews 81 (3) (2001) 1305-1352. doi:10.1152/physrev.2001.81.3.1305.

[27] T. Ren, W. He, D. Kemp, Reticular lamina and basilar membrane vibrations in living mouse cochleae, Proceedings of the National Academy of Sciences 113 (35) (2016) 9910-9915. doi:10.1073/pnas.1607428113.

[28] E. de Boer, R. MacKay, Reflections on reflections, The Journal of the Acoustical Society of America 67 (3) (1980) 882-890. doi:10.1121/1.383968.

[29] C. A. Shera, Mechanisms of Mammalian Otoacoustic Emission and their Implications for the Clinical Utility of Otoacoustic Emissions, Ear and Hearing 25 (2) (2004) 86-97. doi:10.1097/01.AUD.0000121200.90211.83.

[30] A. Sasmal, K. Grosh, Unified cochlear model for low- And high-frequency mammalian hearing, Proceedings of the National Academy of Sciences of the United States of America (2019). doi:10.1073/pnas.1900695116.

[31] J. Tonndorf, Fluid Motion in Cochlear Models, The Journal of the Acoustical Society of America (1957). doi:10.1121/1.1908965.

[32] R. Z. Gan, B. P. Reeves, X. Wang, Modeling of Sound Transmission from Ear Canal to Cochlea, Annals of Biomedical Engineering 35 (12) (2007) 2180-2195. doi:10.1007/s10439-007-9366-y.

[33] M. J. Rapson, T. J. Hamilton, J. C. Tapson, On the fluid-structure interaction in the cochlea, The Journal of the Acoustical Society of America (2014). doi:10.1121/1.4883382. 
[34] W. S. Rhode, C. D. Geisler, Measurement of the Amplitude and Phase of Vibration of the Basilar Membrane Using the Mössbauer Effect, The Journal of the Acoustical Society of America 47 (1A) (1970) 60-60. doi:10.1121/1.1974630.

[35] A. Karlos, Wave propagation in non-uniform waveguides, Ph.D. thesis, University of Southampton (2020).

URL http://eprints.soton.ac.uk/id/eprint/441961

[36] A. Karlos, S. J. Elliott, Cochlea-inspired design of an acoustic rainbow sensor with a smoothly varying frequency response, Scientific Reports 10 (1) (2020) 10803. doi:10.1038/s41598-020-67608-z.

[37] R. H. Ghose, Microwave Circuit Theory and Analysis, McGraw-Hill Book Company, Inc., United States of America, 1963. 